\title{
Theoretical Modeling of Thermodynamic and Mechanical Properties of the Pure Components of Ti and Zr Based Alloys Using the Exact Muffin-Tin Orbitals Method
}

Igor Abrikosov, A. Yu Nikonov, A. V. Ponomareva, A. I. Dmitriev and S. A. Barannikova

\author{
Linköping University Post Print
}

\section{Tweet}

N.B.: When citing this work, cite the original article.

The original publication is available at www.springerlink.com:

Igor Abrikosov, A. Yu Nikonov, A. V. Ponomareva, A. I. Dmitriev and S. A. Barannikova, Theoretical Modeling of Thermodynamic and Mechanical Properties of the Pure Components of $\mathrm{Ti}$ and $\mathrm{Zr}$ Based Alloys Using the Exact Muffin-Tin Orbitals Method, 2014, Russian Physics Journal, (56), 9, 1030-1038.

http://dx.doi.org/10.1007/s11182-014-0136-7

Copyright: Springer Verlag (Germany) http://www.springerlink.com/?MUD=MP

Postprint available at: Linköping University Electronic Press

http://urn.kb.se/resolve?urn=urn:nbn:se:liu:diva-105042 
${ }^{1}$ Department of Physics, Chemistry and Biology of Linköping University, Linköping, Sweden; ${ }^{2}$ Institute of the Physics of Strength and Materials Science, Siberian Branch of the Russian Academy of Sciences, Tomsk, Russia; ${ }^{3}$ National University of Science and Technology "MISIS," Moscow, Russia; ${ }^{4}$ National Research Tomsk State University, Tomsk, Russia, e-mail: dmitr@ispms.tsc.ru. Translated from Izvestiya Vysshikh Uchebnykh Zavedenii, Fizika, No. 9, pp. 52-59, September, 2013. Original article submitted June 27, 2013.

\title{
THEORETICAL MODELING OF THERMODYNAMIC AND MECHANICAL PROPERTIES OF THE PURE COMPONENTS OF Ti AND Zr BASED ALLOYS USING THE EXACT MUFFIN-TIN ORBITALS METHOD
}

\author{
I. A. Abrikosov, ${ }^{1}$ A. Yu. Nikonov, ${ }^{2}$ A. V. Ponomareva, ${ }^{3}$ \\ A. I. Dmitriev, ${ }^{2,4}$ S. A. Barannikova ${ }^{2,4}$
}

UDC 539.21

The Exact Muffin-Tin Orbitals (EMTO) method belongs to the third and latest generation of first-principles methods of calculating the electronic structure of materials in the so-called approximation of muffin-tin (MT) orbitals within the framework of the density functional theoryA study has been performed of its applicability for modeling the thermodynamic and mechanical properties of the pure components of $\mathrm{Ti}$ and $\mathrm{Zr}$ based alloys. The total energies of $\mathrm{Ti}, \mathrm{Zr}, \mathrm{Nb}, \mathrm{V}, \mathrm{Mo}$, and $\mathrm{Al}$ are calculated in three crystal structures - face-centered cubic (FCC), body-centered cubic (BCC), and hexagonal close-packed (HCP). For all of these elements and crystal structures, we have calculated the theoretical values of the lattice constants, elastic constants, and equations of state. The stable crystal structures have been determined. In all cases, calculations by the EMTO method predict the correct ground state structure. For stable structures we compared the obtained results with experiment and with calculations using full potential methods. We have demonstrated the reliability of the EMTO method and conclude that its further application for effective modeling of the properties of disordered alloys based on $\mathrm{Ti}$ and $\mathrm{Zr}$ is possible.

Keywords: first-principles calculation of electronic structure, stability of crystal structure, elastic constants, Ti, $\mathrm{Zr}, \mathrm{Nb}, \mathrm{V}, \mathrm{Mo}$, and $\mathrm{Al}$.

\section{INTRODUCTION}

The accelerated development of cutting-edge technologies has substantially increased the need for new materials and new alloys with special properties. In recent years, qualitatively new classes of materials have been developed which are attractive both from the point of view of pure research and gaining an understanding of various fundamental properties and for use in various practical applications. The dominant approach in the search for new materials has been and remains experiment. At the same time, theory has been playing an ever more important role in materials science. Computer modeling in parallel with experiment makes it possible to obtain a much more complete picture of the physical mechanisms determining the conditions of synthesis and also the properties of materials. One of the most impressive events in the quantum theory of the solid state over the last three decades has been the appearance of a new field - computer modeling of the properties of materials from first principles ( $a b$ initio) on the basis of the density functional theory(DFT). However, at its present stage calculations within the framework of DFT continue to be done for materials with an ideal three-dimensional periodic structure since group theory and the Bloch theorem are cornerstone concepts in the theory of electronic structure [1]. Therefore, to model defects of crystal structure, e.g., substitution impurities or chemical disorder, it is necessary to use the so-called supercell method, in which a quasidisordered periodic model is used to describe the disordered structure [2]. Apart from its fundamental limitations, the supercell model is quite cumbersome.

It should be noted that for a theoretical description of alloys with substitutional disorder there is also an alternative approach, described in the Green's function formalism and the coherentpotential approximation (CPA) [1, 2]. 
Usually, CPA is used together with methods for calculating electronic structure based on the MT potential approximation $[1,2]$. The main problem in earlier variants of this approximation was an inexact account of the interstitial contribution to the potential and/or neglect of overlap of MT spheres centered at different atoms. However, in the third-generation method of MT orbitals it has become possible to solve this problem exactly, for which reason this method is called the exact MT orbitals (EMTO) method [3,4]. Realization of CPA on the basis of EMTO with the help of the full charge density technique[5] has made it possible to model successfully an entire spectrum of technologically important systems [4]. The aim of the present work was to investigate the applicability of the EMTO method in the case of modeling of the thermodynamic and mechanical properties of the pure components of $\mathrm{Ti}$ and $\mathrm{Zr}$ based alloys. The choice of materials is explained by the uniqueness of the properties of alloys based on them.

\section{TECHNIQUE FOR CALCULATING THE THERMODYNAMIC AND MECHANICAL PROPERTIES OF MATERIALS USING THE EMTO METHOD}

The theoretical bases of EMTO are described in detail in a number of works [3-5]. The basis set of EMTO wave functions included $s-, p-, d$-, and $f$ - orbitals. To calculate the elastic properties, the integration over the irreducible part of the Brillouin zone was performed using a $31 \times 31 \times 31$ grid of $k$-points in the reciprocal space for cubic lattices, for hexagonal crystals we used $25 \times 25 \times 21,18 \times 18 \times 12$, and $21 \times 21 \times 17$ grids of $k$-points to calculate $C_{66}, C_{44}$, and $C_{S}$, respectively. For the integration over energy in the complex plane we used 24 points over a semicircular contour. The convergence of the energy with respect to the calculation parameters was $10^{-8} \mathrm{Ry}$. Exchange-correlation effects in the electron gas were taken into account within the framework of the generalized gradient approximation (GGA) [6].

The equation of state was determined by calculating the total energy of the material $E\left(V_{\mathrm{at}}\right)$ for the corresponding values of the volume per atom $V_{\text {at }}$. In the given work $E\left(V_{\text {at }}\right)$ was approximated with the help of the modified Morse function [7]. Note that for HCP crystals it was initially necessary to optimize the ratio of the lattice constants $c / a$ for each volume $V_{\text {at }}$ by minimizing the energy $E\left(V_{\text {at }}, c / a\right)$ with respect to $c / a$. The equations of state are constructed with the help of the so-obtained minima of the function $E\left(V_{\mathrm{at}}, c / a\right)$.

The adiabatic elastic constants for single crystals in this work were calculated by first calculating the total energies of the alloy, obtained for a series of volume-conserving $(V=$ const $)$ small deformations in the range of applicability of Hooke's law. Since the energy of the alloy depends on the volume stronger than on the volume conserving distortions, we do not have to take account the change in the energy caused by a change in the volume because of the above mention condition. For cubic crystals there exist three independent elements of the tensor of elastic constants: $C_{11}, C_{12}, C_{44}$, but in a hexagonal crystal there are five independent elastic constants: $C_{11}, C_{12}, C_{13}, C_{33}$, $C_{44}$. The elastic constants were calculated using the equilibrium values of the lattice constants, following the methodology outlined in detail in $[4,8]$.

The criterion of stability of the crystal lattice is derived from the condition that the energy should increase upon any small deformation. For cubic crystals the stability criterion is given by the relations

$$
C_{44}>0, C_{11}>\left|C_{12}\right|, C_{11}+2 C_{12}>0 \text {. }
$$

For a HCP crystal, the stability conditions (besides the positive definite diagonal components of the rigidity tensor $C_{11}, C_{33}, C_{44}, C_{66}$ ) have the form

$$
C_{11}^{2}>C_{12}^{2},\left(C_{11}+C_{12}\right) C_{33}>2 C_{13}^{2}, C_{11} C_{33}>C_{13}^{2} .
$$




\section{CALCULATED RESULTS AND ANALYSIS}

\section{Calculation of the lattice constant}

As was noted in the description of the calculational technique, to determine the thermodynamic properties of HCP metals it is first of all necessary to optimize the total energy over the volume and the c/a ratio. The ideal value of this ratio is 1.633 . However, in the majority of HCP metals there are deviations from the ideal value. Moreover, the possibility exists of the existence of not only a global minimum, but also local minima of the potential energy as in the case with $\mathrm{Zn} \mathrm{[5]} \mathrm{for} \mathrm{example.} \mathrm{In} \mathrm{order} \mathrm{to} \mathrm{avoid} \mathrm{situations} \mathrm{where} \mathrm{the} \mathrm{calculated} \mathrm{value} \mathrm{of} c / a$ erroneously corresponds to a local minimum, it makes sense to vary $c / a$ as well as the volume over a wide enough range.

TABLE 1. Calculated values of the lattice constants $(a)$, the ratio $c / a$, the bulk modulus $(B)$, and the elastic constants $\left(C_{11}, C_{12}, C_{13}, C_{66}, C_{44}\right)$ for $\mathrm{Ti}, \mathrm{Zr}, \mathrm{Mo}, \mathrm{Nb}, \mathrm{V}$, and $\mathrm{Al}$ with the ground state structure

\begin{tabular}{|c|c|c|c|c|c|c|c|c|c|}
\hline Method & $a, \AA$ & $c / a$ & $B, \mathrm{GPa}$ & $C_{11}, \mathrm{GPa}$ & $C_{12}, \mathrm{GPa}$ & $C_{13}, \mathrm{GPa}$ & $C_{33}, \mathrm{GPa}$ & $C_{66}, \mathrm{GPa}$ & $C_{44}, \mathrm{GPa}$ \\
\hline \multicolumn{10}{|c|}{$\mathrm{Ti}, \mathrm{hcp}$} \\
\hline TMTO & 2.93 & 1.608 & 110 & 207 & 60 & 58 & 219 & 74 & 49 \\
\hline VASP & $\begin{array}{l}2.929^{\mathrm{a}} \\
2.931^{\mathrm{b}}\end{array}$ & $\begin{array}{l}1.580^{\mathrm{a}} \\
1.596^{\mathrm{b}}\end{array}$ & & $172^{\mathrm{b}}$ & $82^{b}$ & $75^{\mathrm{b}}$ & $190^{\mathrm{b}}$ & $45^{\mathrm{b}}$ & $45^{\mathrm{b}}$ \\
\hline Exp & $2.95^{\mathrm{c}}$ & $1.586^{\mathrm{c}}$ & $114^{\mathrm{c}}$ & $176^{\mathrm{c}}$ & $87^{\mathrm{c}}$ & $68^{c}$ & $191^{\mathrm{c}}$ & $44.6^{c}$ & $51^{\mathrm{c}}$ \\
\hline \multicolumn{10}{|c|}{$\mathrm{Zr}$, hcp } \\
\hline TMTO & 3.23 & 1.616 & 85 & 155 & 40 & 49 & 197 & 58 & 35 \\
\hline VASP & $\begin{array}{l}3.230^{\mathrm{a}} \\
3.236^{\mathrm{d}}\end{array}$ & $1.606^{\mathrm{a}}$ & $96.0^{\mathrm{d}}$ & $146.7^{\mathrm{d}}$ & $68.5^{\mathrm{d}}$ & $71^{d}$ & $163.3^{\mathrm{d}}$ & & $26^{d}$ \\
\hline Exp & $3.23^{\mathrm{e}}$ & $1.592^{\mathrm{e}}$ & $94^{\mathrm{f}}$ & $144^{\mathrm{f}}$ & $74^{\mathrm{f}}$ & $67^{f}$ & $167^{\mathrm{f}}$ & $35^{\mathrm{f}}$ & $33^{f}$ \\
\hline \multicolumn{10}{|c|}{ Mo, bcc } \\
\hline TMTO & 3.16 & 1 & 263 & 489 & 145 & & & & 119 \\
\hline VASP [9] & 3.178 & & & & & & & & \\
\hline Exp [16] & 3.15 & 1 & 261 & 450 & 173 & & & & 125 \\
\hline \multicolumn{10}{|c|}{$\mathrm{Nb}, \mathrm{bcc}$} \\
\hline TMTO & 3.31 & 1 & 161 & 258 & 113 & & & & 39 \\
\hline VASP [9] & 3.322 & 1.821 & & & & & & & \\
\hline Exp [17] & 3.30 & 1 & 171 & 247 & 133 & & & & 28 \\
\hline \multicolumn{10}{|c|}{$\mathrm{V}, \mathrm{bcc}$} \\
\hline TMTO & 3.00 & 1 & 174 & 281 & 125 & & & & 36 \\
\hline VASP[9] & 2.99 & 1 & & & & & & & \\
\hline $\operatorname{Exp}[18]$ & 3.03 & 1 & 160 & 237 & 121 & & & & 47 \\
\hline \multicolumn{10}{|c|}{$\mathrm{Al}, \mathrm{fcc}$} \\
\hline TMTO & 4.05 & 1 & 80 & 106 & 67 & & & & 52 \\
\hline VASP [9] & 4.048 & 1 & & & & & & & \\
\hline $\operatorname{Exp}[19]$ & 4.00 & 1 & 76 & 107 & 61 & & & & 29 \\
\hline
\end{tabular}

The results of calculation by the EMTO method of changes in the total energy of $\mathrm{HCP} \mathrm{Ti}, \mathrm{Zr}, \mathrm{Nb}, \mathrm{V}, \mathrm{Mo}$, and $\mathrm{Al}$ as functions of these parameters showed that for all systems examined in this work there is a unique energy minimum as a function of $V_{\text {at }}$ and cla. Since the main problem is checking the accuracy of the EMTO method for the pure elements of $\mathrm{Ti}$ and $\mathrm{Zr}$ based alloys, theoretical optimized and experimental values of the $a$ parameters and the $c / a$ ratios for these elements are given in Table 1. In addition, the accuracy of the method was estimated by comparing with the results of calculations performed using the projector augmented wave (PAW) method using the software package 
VASP (Vienna ab-initio Simulation Package) in [9]. This method can be considered to be the most accurate method available at present; however, it is too cumbersome for high-output calculation of properties of substitutional alloys since in this case it is necessary to resort to the supercell method to model disorder effects [2]. In the EMTO method, modeling of disorder effects is realized within the framework of the numerically efficient coherent potential approximation (CPA) [2, 5], which makes it possible to significantly reduce the computational costs.

Comparison of values of the lattice constants calculated by the EMTO method with experimental results shows that the accuracy of the theory stands at $\sim 1 \%$, which is typical accuracy for the gradient corrections approximation in DFT. Comparing the results of two different theoretical methods, it becomes clear that for $\mathrm{Ti}$ and $\mathrm{Zr}$ the agreement is close to ideal. However, calculations performed for elements that are unstable in the HCP structure, i.e., Mo, Nb, and $\mathrm{V}$, showed that the agreement, while remaining satisfactory, is somewhat degraded, especially for the parameter $c / a$. The reason for this lies in the fact that for the first group of elements there is a clearly expressed, sharp minimum of the potential energy while for the second group the surface is flatter. In this regard, for $\mathrm{Ti}, \mathrm{Zr}$, and $\mathrm{Al}$ the value of $c / a$ is close to ideal, and these elements either are stable in the HCP phase or transition into it under high pressures, as is the case for $\mathrm{Al}$ [15]. On the other hand, for $\mathrm{Mo}, \mathrm{Nb}$, and $\mathrm{V}$ the value of $c / a$ deviates strongly from its ideal value, and their transition to the HCP phase has so far not been experimentally demonstrated. Moreover, these elements are mechanically unstable; therefore, the potential energy surface for them is more complex, which also leads to greater sensitivity of the results to the details of the calculation.

It should be noted, however, that a change in the volume leads to markedly stronger variations in the energy in comparison with the effect of varying $c / a$. The usual accuracy of calculation within the framework of DFT of such very important characteristics of alloys as, for example, the mixing enthalpy, stands at $\sim 1-2 \mathrm{mRy} / \mathrm{atom}$. Therefore, to determine the equilibrium values of the total energy, optimization over volume is of definitive importance, whereas the calculation of the ratio cla tolerates a certain error. In this sense, the accuracy of the EMTO method should be seen as satisfactory even for a situation that is so challenging to model as optimization of the lattice constants of mechanically unstable systems.

Estimating the accuracy of calculation of the lattice constants of cubic crystals by the EMTO method (see Table 1), it is possible to conclude that in comparison with the experimental data available for stable structures, the error is again found within the limits of $1 \%$. Comparing the results of various theoretical calculations amongst themselves, it is possible to conclude that the agreement is even better than for HCP metals. This can be explained by the fact that optimization in the given case is performed over only one parameter $a$.

\section{Calculation of the elastic modulus}

Values of the bulk moduli for the metals considered here in their stable crystal structures are listed in Table 1. Analyzing the bulk moduli calculated by the EMTO method, it is important to point out that, as is well known, the accuracy of first-principles calculations of the elastic constants is lower than for the lattice constants, and stands at about $10 \%$. The reason for this consists in the fact that the elastic constants are determined by the second derivatives of the energy with respect to the volume, and the volume itself enters directly into the expression for the bulk modulus. For an accuracy of calculation of the lattice constant of $1 \%$ the error in determining the volume stands at $\sim 3 \%$, which translates into a calculational error for $B$ of $\sim 10 \%$. However, it should be noted that only for two metals, BCC V and $\mathrm{HCP} \mathrm{Zr}$, does the error in our calculations approach this value. The accuracy of calculation for the remaining stable phases of the metals considered in the present work is markedly higher. The good agreement between the various theoretical calculations should also be noted.

Perhaps, a more complex test for the EMTO method would be to calculate the elastic constants of single crystals of the metals considered in this work since in the calculation of the self-consistent charge density the spherical cells approximation (SCA) for the potential is still used, which gives an unsatisfactory description of the distortions of the crystalline lattice (with the exception of uniform compression, as in the case of the calculation of the bulk modulus). The elastic constants obtained with the help of the EMTO method are given in Table 1.

It can be seen that on the whole the calculations reproduce well the behavior of the elastic constants observed in experiment. The obtained results can be divided into two groups: those with comparatively large (>100 GPa) values 
and those with comparatively small $(<100 \mathrm{GPa})$ values. For the first group the error in the calculations is found within the limits of $20 \%$, but in many cases the agreement is better. For the second group the absolute difference between theory and experiment remains of the same order as for the first group $(\sim 30 \mathrm{GPa})$, but due to the small values of the elastic constants the relative error can be quite large. It can also be seen that the use of full-potential methods allows one to model more reliably the elastic constants from first principles although for small values, also in this case, one can expect an increase in the relative error. The increase in the relative error of the EMTO method for lower numerical values of the elastic constants is explained by the fact that changes in the total energy as functions of the magnitude of the deformation become very small and very high accuracy is needed to describe them.

It should, however, be noted that the elastic constants of single crystals find limited application in the development of technological materials, and of greatest importance on this plane are rather their elastic moduli. The Reuss (R), Voigt (V), and Hill (H) average values of the Young modulus $(E)$, the shear modulus $(G)$, and the Poisson ratio $(v)$ for the elements considered in this work in their stable crystal structures are given in Table 2. It can be seen that for cubic crystals the errors in the calculation of the elements of the tensor of elastic constants of single crystals are to a great extent reduced, and for polycrystalline elastic constants the agreement with experiment turns out to be entirely satisfactory. Therefore, it can be expected that the use of the EMTO method will be useful in the development of new alloys based on $\mathrm{Ti}$ and $\mathrm{Zr}$ since one can expect a correct description of the concentration dependence of such important mechanical properties as the Young modulus, which determines to a great extent the strength of a material, or the ratio of the shear modulus to the elasticity modulus $G / B$, often used as a characteristic of plastic or brittle behavior of materials. For hexagonal alloys based on $\mathrm{Ti}$ and $\mathrm{Zr}$ the values of the elastic constants calculated by the EMTO method should be used with greater caution.

TABLE 2. The Reuss (R), Voigt (V), and Hill $(\mathrm{H})$ average values of the Young modulus $(E)$, the shear modulus $(G)$, and the Poisson ratio $(v)$ for the investigated metals in their stable crystal structures

\begin{tabular}{|c|c|c|c|c|c|c|c|c|c|}
\hline \multirow{2}{*}{ Method } & \multicolumn{3}{|c|}{$E, \mathrm{GPa}$} & \multicolumn{3}{|c|}{$G, \mathrm{GPa}$} & \multicolumn{3}{|c|}{$v$} \\
\hline & $\mathrm{R}$ & $\mathrm{V}$ & $\mathrm{H}$ & $\mathrm{R}$ & $\mathrm{V}$ & $\mathrm{H}$ & $\mathrm{R}$ & $\mathrm{V}$ & $\mathrm{H}$ \\
\hline $\begin{array}{l}\text { Mo, bcc, } \\
\text { TMTO }\end{array}$ & 348 & 358 & 352 & 136 & 140 & 138 & 0.279 & 0.274 & 0.277 \\
\hline $\begin{array}{c}\text { Mo, Exp } \\
{[18]}\end{array}$ & \multicolumn{3}{|c|}{336} & \multicolumn{3}{|c|}{130} & \multicolumn{3}{|c|}{0.29} \\
\hline $\begin{array}{c}\text { Nb, bcc, } \\
\text { TMTO }\end{array}$ & 133 & 143 & 138 & 48.9 & 53 & 51 & 0.374 & 0.363 & 0.37 \\
\hline $\begin{array}{l}\text { Exp [20], } \\
T=298 \mathrm{~K}\end{array}$ & \multicolumn{3}{|c|}{119} & \multicolumn{3}{|c|}{43} & \multicolumn{3}{|c|}{0.37} \\
\hline $\begin{array}{l}\text { V, bcc, } \\
\text { TMTO }\end{array}$ & 126.8 & 145 & 136 & 45.9 & 53.2 & 50 & 0.38 & 0.36 & 0.37 \\
\hline $\begin{array}{l}\text { Exp [20], } \\
T=298 \mathrm{~K}\end{array}$ & \multicolumn{3}{|c|}{137} & \multicolumn{3}{|c|}{49} & \multicolumn{3}{|c|}{0.36} \\
\hline $\begin{array}{l}\text { Al, fcc, } \\
\text { TMTO }\end{array}$ & 84 & 102 & 93 & 31 & 40 & 35 & 0.32 & 0.28 & 0.31 \\
\hline $\begin{array}{l}\text { Exp [20], } \\
T=298 \mathrm{~K}\end{array}$ & \multicolumn{3}{|c|}{70} & \multicolumn{3}{|c|}{26} & \multicolumn{3}{|c|}{0.35} \\
\hline $\begin{array}{l}\text { Ti, hcp, } \\
\text { TMTO }\end{array}$ & 154 & 159 & 157 & 64 & 62.8 & 63 & 0,23 & 0.28 & 0.26 \\
\hline $\begin{array}{l}\text { Exp [20], } \\
T=298 \mathrm{~K}\end{array}$ & \multicolumn{3}{|c|}{103} & \multicolumn{3}{|c|}{38} & \multicolumn{3}{|c|}{0.36} \\
\hline $\begin{array}{c}\text { Zr, hcp, } \\
\text { TMTO }\end{array}$ & 119 & 127 & 123 & 49 & 51 & 50 & 0.22 & 0.27 & 0.25 \\
\hline $\begin{array}{l}E x p[20], \\
T=298 \mathrm{~K}\end{array}$ & \multicolumn{3}{|c|}{68} & \multicolumn{3}{|c|}{25} & \multicolumn{3}{|c|}{0.37} \\
\hline
\end{tabular}


Calculation of the elastic constants allows us to answer still one more important question: is a given crystal structure mechanically stable. Using the elastic constants from Table 1 and the criteria of mechanical stability, it is possible to conclude that all the elements considered in this work are mechanically stable in crystal structures corresponding to their experimental ground state. At the same time, our calculations have shown that the FCC and HCP phases of $\mathrm{V}, \mathrm{Mo}$, and $\mathrm{Nb}$, and also $\mathrm{BCC} \mathrm{Al}$ are mechanically unstable. This means that these phases cannot exist at $T=$ $0 \mathrm{~K}$ since long-wavelength phonon vibrations corresponding to the $\Gamma$ point in the Brillouin zone immediately destabilize them.

It is important to underline that the phases that are mechanically and/or dynamically unstable at temperature $T=0 \mathrm{~K}$ and pressure $P=0 \mathrm{GPa}$ can become metastable and even thermodynamically stable with increase of the temperature and/or under pressure. Characteristic examples here are precisely the $\mathrm{BCC}$ phases of $\mathrm{Ti}$ and $\mathrm{Zr}$ observed in experiment. Stabilization of these phases is possible due to the effects of anharmonicity; therefore, a knowledge of the thermodynamic properties of such phases is in anyhow very important, for example, in the modeling of phase equilibria. However, in this case the thermodynamic properties can depend strongly on temperature, which must be kept in mind during the modeling.

\section{Dependence of the potential energy on the type of crystalline lattice}

The concept of stability of a crystalline lattice, defined as the difference in the Gibbs free energies of the chemically equivalent materials with different crystal structure, was ground-laying in the creation of the powerful thermodynamic tool for calculating phase diagrams known as CALPHAD (CALculations of PHAse Diagrams) [9]. It is important to note that this concept includes within itself not only experimentally observed structures but also structures not observed in experiment. At first, the lack of experimental information was compensated by extrapolation of the phase diagrams. With the development of computer modeling it became possible to calculate these quantities from first principles.

TABLE 3. Calculated values of the difference of potential energies (in Ry/atom) between the structures FCC $-\mathrm{BCC}$ and $\mathrm{HCP}$ - BCC for $\mathrm{Al}, \mathrm{Mo}, \mathrm{Nb}, \mathrm{Ti}, \mathrm{V}$, and $\mathrm{Zr}$

\begin{tabular}{c|c|c|c|c|c}
\hline \hline Element & FCC - BCC & $\begin{array}{c}\text { FCC }- \text { BCC, } \\
\text { VASP [9] }\end{array}$ & HCP - BCC & $\begin{array}{c}\text { HCP }- \text { BCC, } \\
\text { VASP [9] }\end{array}$ & Ground state \\
\hline $\mathrm{Al}$ & -0.0050 & -0.0070 & -0.0027 & -0.0048 & FCC \\
\hline $\mathrm{Mo}$ & 0.0350 & 0.0295 & 0.0386 & 0.0304 & BCC \\
\hline $\mathrm{Nb}$ & 0.0261 & 0.0238 & 0.0281 & 0.0214 & $\mathrm{BCC}$ \\
\hline $\mathrm{Ti}$ & -0.0016 & -0.0036 & -0.0059 & -0.0078 & $\mathrm{HCP}$ \\
\hline $\mathrm{V}$ & 0.0199 & 0.0182 & 0.0234 & 0.0186 & $\mathrm{BCC}$ \\
\hline $\mathrm{Zr}$ & -0.0004 & -0.0027 & -0.0042 & -0.0056 & $\mathrm{HCP}$ \\
\hline
\end{tabular}

Table 3 summarizes the results of calculations of the potential energy difference between the FCC - BCC and $\mathrm{HCP}-\mathrm{BCC}$ structures. It can be seen that at their equilibrium volumes (at zero pressure) $\mathrm{Ti}$ and $\mathrm{Zr}$ are stable in the $\mathrm{HCP}$ structure, $\mathrm{Nb}, \mathrm{V}$, and Mo are stable in the $\mathrm{BCC}$ structure while $\mathrm{Al}$ is stable in the FCC structure. Thus, calculation finds itself to be in complete agreement with experiment. Analyzing the results obtained using the EMTO method and the software package VASP [9], it can be seen that on the whole there is good qualitative agreement between them. The greatest difference occurs in the calculation for Mo. This is explained by the fact that the difference in structural energies of the transition metals is determined by filling of the valence $d$-band, or the so-called electron density. Mo is found near a deep minimum corresponding to maximum stability of the BCC phase [9], where minimal variations of the parameters of the calculation lead to a large spread in the results. Indeed, the data in the literature on the difference in structural energies FCC - BCC in Mo vary over quite a wide range. 
Equation of state and stability of the crystalline lattice under pressure

Figure 1 plots the calculated equations of state, i.e., the dependence of the volume per atom $V_{\text {at }}$ on the pressure $P$, for $\mathrm{Ti}, \mathrm{Zr}, \mathrm{Nb}, \mathrm{V}, \mathrm{Mo}$, and $\mathrm{Al}$ in the three crystal structures considered in this work. Since the equilibrium volume is determined by the EMTO method with quite high accuracy, good agreement with experiment can also be expected for the equation of state. This is indeed the case for the absolute majority of systems considered here. Thus, we can conclude that the EMTO method describes well the behavior of the total energy of the system as a function of the volume (or the pressure) and can be used to develop new technologies in connection with the production of pressure treated alloys.

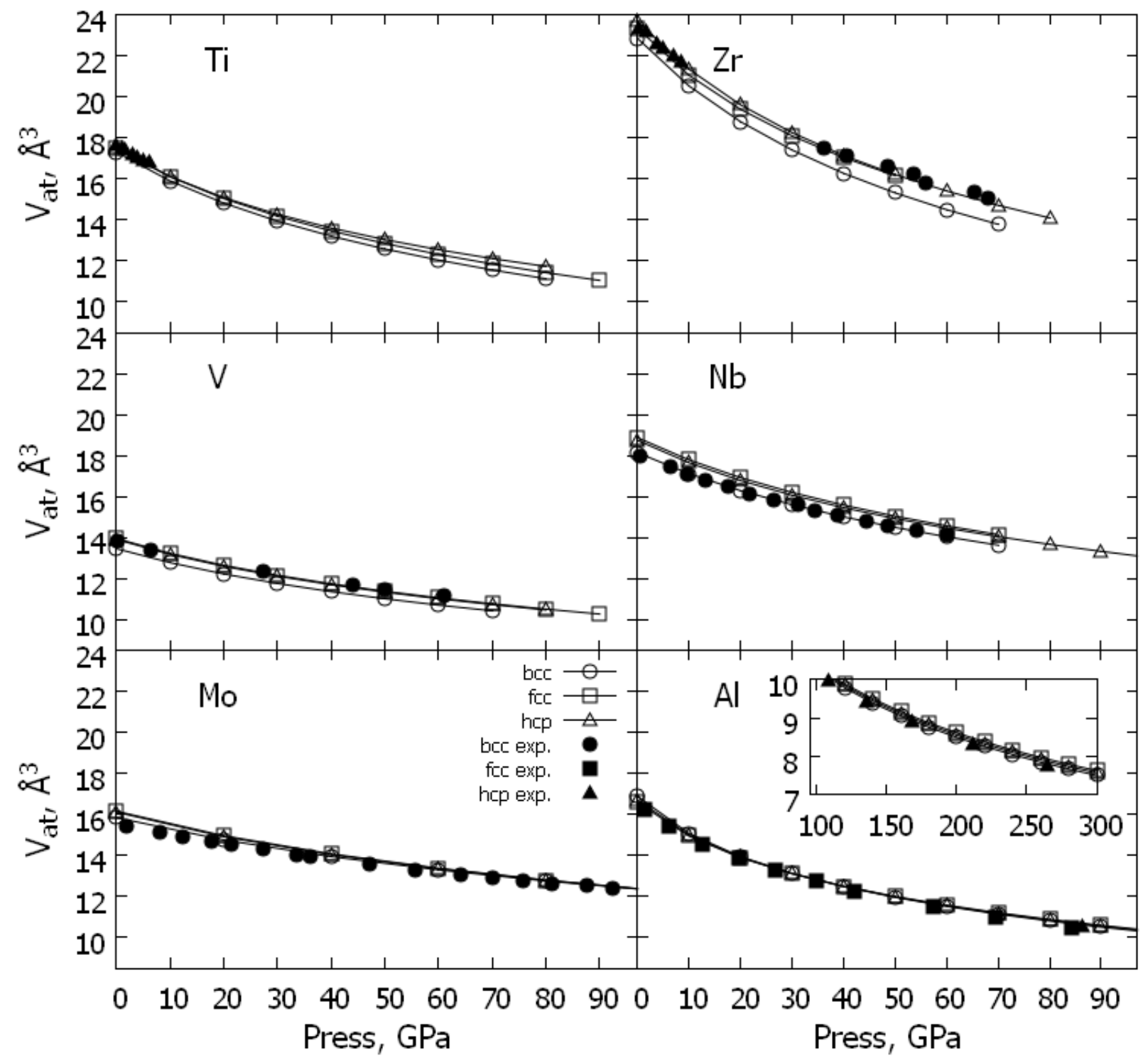

Fig. 1. Calculated equations of state of $\mathrm{Ti}, \mathrm{Zr}, \mathrm{Nb}, \mathrm{V}, \mathrm{Mo}$, and $\mathrm{Al}$ in three crystal structures: $\mathrm{FCC}$ (open squares), $\mathrm{BCC}$ (open circles), and HCP (open triangles). The corresponding experimental values are represented by the filled symbols. HCP Ti [11]; BCC V [21]; BCC Mo [22]; HCP and BCC Zr [23]; BCC Nb [23], FCC Al [24], HCP Al [15]. Inset in the $\mathrm{Al}$ panel gives a magnified picture of the region of the $\mathrm{FCC}-\mathrm{HCP}$ phase transition.
ANNOTATION:
Abscissa ( $\times 2)$ :
$P, \mathrm{GPa}$
Ordinate $(\times 3)$ :
$V_{\text {at }}, \AA^{3}$ 
In this regard, the comparatively poor agreement between the theoretical and experimental equations of state for $\mathrm{BCC} \mathrm{Zr}$ is noteworthy. However, there is nothing remarkable about this. The theoretical calculation was carried out for $T=0 \mathrm{~K}$ whereas experimentally BCC Zr stabilizes only at high temperatures. BCC $\mathrm{Zr}$ is dynamically unstable, and its potential energy depends strongly on temperature. As was recently demonstrated by Hellman et al. [25], taking temperature effects into account in the modeling of the equation of state of $\mathrm{Zr}$ leads to roughly a $5 \%$ growth in the volume for fixed pressure. For the HCP phase, which is stable at low temperatures, and where the effects of anharmonicity are small, good agreement of calculation at $T=0 \mathrm{~K}$ can be expected with experiment, which in fact is seen in Fig. 1.

Note also that with increasing pressure the correlations between the potential energies of the phases considered in this work vary for $\mathrm{Ti}, \mathrm{Zr}$, and $\mathrm{Al}$. For $\mathrm{Al}$ the $\mathrm{HCP}$ phase becomes the most stable phase whereas for $\mathrm{Ti}$ and $\mathrm{Zr}$ the BCC phase becomes the most stable phase. The FCC - HCP transition in Al is indeed detected in experiment at a pressure of $\sim 217 \mathrm{GPa}$. This result is found to be in very good agreement with our theoretical calculation. For $\mathrm{Zr}$ and $\mathrm{Ti}$ the situation is somewhat more complicated. Since the effect of pressure in the case of the transition metals leads to a growth of the electron density in the $d$ band due to charge transfer between $s p$ - and $d$-electrons, for these metals one can indeed expect stabilization of the BCC phase in comparison with the HCP phase [13]. However, in experiment this transition does not take place directly, but is realized as a series of transitions, including the $\omega$-phase [13]. The latter phase was not considered in this work; therefore, it is impossible to compare the theoretically obtained transition pressure with experiment. However, it is obvious that the EMTO method correctly describes the tendency of the change in stability of the crystalline lattice under pressure.

\section{CONCLUSIONS}

In the present work, using the numerically efficient EMTO method, we have modeled from first principles the behavior of the metallic elements $\mathrm{Ti}, \mathrm{Zr}, \mathrm{V}, \mathrm{Nb}, \mathrm{Mo}$, and $\mathrm{Al}$ with the aim of determining the adequacy of the approximations on which the EMTO method is based and investigating its potential for modeling $\mathrm{Ti}$ and $\mathrm{Zr}$ based alloys. The equations of state were calculated and the elastic constants were determined. The results of these calculations showed that in all cases the EMTO method predicts the correct crystal structure of the ground state: HCP for $\mathrm{Ti}$ and $\mathrm{Zr}, \mathrm{BCC}$ for $\mathrm{V}, \mathrm{Mo}$, and $\mathrm{Nb}$, and FCC for Al. For the ground-state structures we obtained good agreement of the equilibrium lattice constants with the experimental data. For the non-equilibrium structures the predicted lattice constants can be initial parameters for the construction of next-level thermodynamic models within the framework of multilevel modeling.

The elastic constants of $\mathrm{Ti}, \mathrm{Zr}, \mathrm{V}, \mathrm{Nb}, \mathrm{Mo}$, and $\mathrm{Al}$ were calculated for the ground-state structure, and also from non-equilibrium crystal structures which are of interest for further study. For the majority of the elastic constants we obtained good agreement with the experimental data. However, it turned out that the relative accuracy of the EMTO method in the calculation of the elastic constants decreases with decrease of the absolute magnitude of these constants. In the calculation of the non-equilibrium structures it was determined that some of them are mechanically unstable, which is expressed, for example, in negative values of the elastic constants $C_{44}$ and $C_{66}$ for Mo, $\mathrm{V}$, and $\mathrm{Nb}$ in the HCP structure. This result does not contradict experiment since it is well known that the indicated elements cannot be stabilized in the given structures under normal conditions. In the calculation of the elastic constants of a polycrystal for the elements with a cubic lattice, there takes place a mutual compensation of the errors due to averaging, and the accuracy of the prediction by the EMTO method of the elastic constants, which are so important from the technological point of view, turns out to be quite high, similar to the case with the Young modulus.

The results of this work prove that at the present time it is possible in the calculation of the properties of materials from first principles to obtain a level of accuracy that is comparable with that obtained in experiment. The proposed methods allow one to predict the behavior of designed materials under experimental conditions, for example, under ultrahighpressures. These data are extraordinarily helpful in predicting the mechanical properties of new materials and can be brought to bear in the scientifically based design of new alloys based on $\mathrm{Ti}$ and $\mathrm{Zr}$. In addition, calculations from first principles enable a deeper analysis into the essence of physical phenomena taking place in the investigated materials. 
This work was performed within the framework of the Federal Target Program "Scientific and ScientificPedagogical Personnel of Innovative Russia" during the timeframe 2009-2013 pursuant to the measure: 1.5 "Support of Scientific Research Performed by Collectives under the Guidance of Invited Researchers" (Agreement No. 14.B37.21.0890 commencing September 10, 2012) and with partial financial support of the Russian Foundation for Basic Research (Project No. 13-02-00606 a).

\section{REFERENCES}

1. R. M. Martin, Electronic structure. Basic Theory and Practical Methods, Cambridge Univ. Press, Cambridge (2004).

2. A. V. Ruban and I. A. Abrikosov, Rep. Prog. Phys., 71, 046501 (2008).

3. O. K. Andersen, O. Jepsen, and G. Krier, in: Lectures on Methods of Electronic Structure Calculations, edited by V. Kumar, O. Andersen, and A. Mookerjee, World Scientific Publishing Co., Singapore (1994), pp. 63-124.

4. L. Vitos, Computational Quantum Mechanics for Materials Engineers, Springer Verlag, Berlin (2007).

5. L. Vitos, I. A. Abrikosov, and B. Johansson, Phys. Rev. Lett., 87, 156401 (2001).

6. J.P. Perdew, K. Burke, and M. Ernzerhof, Phys. Rev. Lett., 77, 3865 (1996).

7. V. L. Morruzi, J. F. Janak, and K. Schwartz, Phys. Rev., B37, 790 (1988).

8. C. Asker, L. Vitos, and I. A. Abrikosov, Phys. Rev., B79, 214112 (2009).

9. Y. Wang, S. Curtarolo, C. Jiang, et al., CALPHAD: Comput. Coupling Phase Diagrams Thermochem., 28, 79 (2004).

10. R. G. Hennig, T. J. Lenosky, D. R. Trinkle, et al., Phys. Rev., B78, 054121 (2008).

11. J. Z. Zhang, Y. S. Zhao, R. S. Hixson, et al., Phys. Rev., B78, 054119 (2008).

12. $\quad$ B.-T. Wang, P. Zhang, H.-Y. Liu, et al., J. Appl. Phys., 109, 063514 (2011).

13. Y. Zhao, J. Zhang, C. Pantea, et al., Phys. Rev., B71, 184119 (2005).

14. E. A. Brandes, editor, Smithells Metals Reference Book, Butterworth, London (1983).

15. Y. Akahama, M. Nishimura, K. Kinoshita K., et al., Phys. Rev. Lett., 96, 045505 (2006).

16. F. H. Featherstone and J. R. Neighbours, Phys. Rev. 130, No. 4, 1324 (1963).

17. W. E. Hubbell and F. R. Brotzen, J. Appl. Phys., 43, 3306 (1972).

18. D. I. Bolef, R. E. Smith, and J. G. Miller, Phys. Rev., B3, 4100 (1971).

19. G. Simmons and H. Wang, Single Crystal Elastic Constants and Calculated Aggregate Properties: A Handbook, Second Edition, MIT Press, Cambridge, MA(1971).

20. I. N. Frantsevich, F. F. Voronov, and S. A. Bakuta, Elastic Constants and Elastic Moduli of Metals and Nonmetals [in Russian], Naukova Dumka, Kiev (1982).

21. Y. Ding, R. Ahuja, J. Shu, et al., Phys. Rev. Lett., 98, 085502 (2007).

22. A. Dewaele, M. Torrent, P. Loubeyre, and M. Mezouar, Phys. Rev., B78, 104102 (2008).

23. Y. Akahama, M. Kobayashi, and H. Kawamura, J. Phys. Soc. Jpn., 60, 3211 (1991).

24. A. Dewaele, P. Loubeyre, and M. Mezouar, Phys. Rev., B70, 094112 (2004).

25. O. Hellman, P. Steneteg, I. A. Abrikosov, and S. I. Simak, Phys. Rev., B87, 104111 (2013). 